\title{
Germanica
}

GERMANICA $20 \mid 1997$

\section{L'autobiographie moderne}

\section{Avant-propos}

\section{Anne Lagny}

\section{OpenEdition}

Journals

Édition électronique

URL : http://journals.openedition.org/germanica/1916

DOI : 10.4000/germanica. 1916

ISSN : 2107-0784

\section{Éditeur}

Université de Lille

\section{Édition imprimée}

Date de publication : 30 juin 1997

Pagination : 11-13

ISBN : 9782098426320

ISSN : 0984-2632

\section{Référence électronique}

Anne Lagny, «Avant-propos », Germanica [En ligne], 20 | 1997, mis en ligne le 24 juin 2013, consulté le 06 octobre 2020. URL : http://journals.openedition.org/germanica/1916 ; DOI : https://doi.org/ 10.4000/germanica.1916

Ce document a été généré automatiquement le 6 octobre 2020.

(c) Tous droits réservés 


\section{Avant-propos}

\section{Anne Lagny}

1 Pourquoi un numéro thématique sur les autobiographies de langue germanique au $\mathrm{XX}^{\mathrm{e}}$ siècle ? On pourrait répondre brièvement : à un monde en crise répond une crise du genre autobiographique, et ce sont quelques-unes des différentes issues trouvées à cette crise par des écrivains allemands, autrichiens, suédois que nous avons voulu présenter dans ce volume.

Le genre en effet a déjà une longue histoire derrière lui lorsque s'ouvre le siècle. Qu'on le fasse remonter à l'Antiquité comme Georg Misch ou Georges Gusdorf, ou seulement à Rousseau comme Philippe Lejeune, il a constitué des formes canoniques de récit de la vie dont Goethe, avec Dichtung und Wahrheit, constitue l'une des références majeures, en unissant intimement dans une même œuvre la formation de l'individu et les mutations d'une époque. Cela suppose que l'évolution de l'individu est déchiffrable et qu'elle s'inscrit de façon signifiante dans l'époque, qu'elle représente. Cela suppose aussi que l'époque à son tour joue un rôle dans l'histoire et que ce rôle peut se saisir dans le récit d'une vie humaine.

3 Un siècle qui a connu deux guerres mondiales et tant d'autres bouleversements ne pouvait que remettre ces certitudes en question : les individus ont pu y paraître broyés par l'Histoire, des mondes culturels entiers disparaître sans laisser de trace, et surtout les vies individuelles avec leurs ruptures, leurs combats et leurs sacrifices, ont pu sembler dépourvues de sens. Dans un tel univers, est-il encore possible de concevoir un projet autobiographique, à défaut d'édifier une autobiographie?

4 On ne trouvera guère, dans ces œuvres, l'ambition ou la certitude d'embrasser la totalité d'une vie humaine comme un «modèle » d'itinéraire inscrit dans un système de normes philosophiques ou religieuses. Mais paradoxalement, la remise en cause du sujet laisse subsister la dimension de l'individualité, et donc la possibilité d'une écriture du moi. A défaut de se poser en représentant exemplaire de l'époque, l'écrivain peut fonder la légitimité de son entreprise sur la singularité de son expérience et la particularité de sa perspective, entre les deux pôles que sont la recherche d'une identité et la mémoire d'une époque. 
5 L'autobiographie, à l'origine de laquelle on trouve bien souvent « l'appel de l'écrit », la médiation ambiguë du livre (Gilbert Ravy) demeure l'instrument privilégié de la quête de l'identité personnelle. Elle est le lieu où l'auteur entreprend d'élucider, pour luimême et pour d'autres, ce qui est plus que jamais «sa " vérité individuelle, parfois dévoyée en "apologie personnelle", au prix de la négation de la réalité, comme l'enseigne le cas de Jan Myrdal (Elena Balzamo). Mais qu'elle soit centrée sur un moi à la recherche de repères existentiels, comme chez Handke (Brigitte Desbrière), ou qu'elle soit décentrée du moi, orientée vers «l'observation» aiguë de ses contemporains, qui rapproche Canetti des mémorialistes classiques (Rolf Wintermeyer), l'autobiographie moderne est en tout cas fondée sur la consistance d'un vécu singulier, dans sa particularité même. Une attention nouvelle se porte sur l'efficace d'une écriture qui met en jeu celui qui écrit, dans l'instant où il écrit, et prolonge ou pose les questions en suspens dans la vie. Au centre, celle de l'identité, qui n'est pas toujours accessible par la voie de la remémoration, mais se construit dans le présent de l'écriture comme c'est le cas pour Franz Fühmann (Anne Lagny).

6 Le souci de Gottfried Benn de préserver la dimension de la "poésie» de la compromission dans une époque est une exception : il témoigne au fond de l'absence de véritable projet autobiographique (Rammon Reimann). La majorité des autobiographies envisagées ici, postérieures à la Seconde Guerre mondiale, sont le fait d'auteurs qui ne peuvent se définir qu'en revenant sur une époque qui a marqué leur évolution de manière catastrophique et pour laquelle manquent toujours des modèles d'explication satisfaisants. Pour certains, tels Ludwig Harig, Max von der Grün, Peter Rühmkorf, ... l'autobiographie correspond à la tâche de reconstituer a posteriori la phase manquante d'une évolution entravée ou pervertie par des déterminations trop pesantes (Kerstin Adam). Dieter Wellershoff partage avec ses contemporains l'expérience de la " perte du sens objectif », qui consacre le clivage entre l'expérience individuelle et l'événement historique et le renvoie à son intériorité comme seule réalité fiable. La réduction extrême du champ de vision qui en résulte définit les conditions d'un nouveau réalisme, celui d'un témoignage fragmentaire, mais authentique, qui devient le correctif indispensable des thèses générales de l'historien (Gérard Laudin). Une autobiographie comme le Nachruf de Stefan Heym s'inscrit modestement, en dépit de son ampleur, dans le suspens d'une "pause réflexive ", qui permet de réserver la part de l'interrogation et du doute dans un itinéraire qui ne se donne pas comme achevé (Brigitte Hocke).

7 Comment rendre une expérience particulière accessible à d'autres, et de quelle autorité se prévaloir ? Cette question commune prend un relief particulier dans le cas de Manès Sperber, investi de la mission de transmettre la mémoire du monde disparu du shtetl, dont il est l'un des seuls survivants. Il choisit de s'imposer une forme de distance, seule capable pour lui de faire passer son témoignage personnel dans le patrimoine de la mémoire collective (Anne-Marie Corbin-Schuffels) 